\title{
412. Totale Pankreatektomie bei Nesidioblastose - Eine therapeutische Alternative mit Langzeiterfolg
}

\author{
P. Dohrmann, K. Kruse, W. Mengel, H. Rohwedder und H. Hamelmann \\ Chirurgische Universitätsklinik, Hospitalstraße 40, D-2300 Kiel
}

Total Pancreatectomy in Nesidioblastosis: A Therapeutic Alternative With Long-Lasting Favourable Results

\begin{abstract}
Summary. A newborn had hyperinsulinism with frequent seizures that did not respond to conservative therapy. Nesidioblastosis was the final diagnosis. Hyerinsulinism persisted after subtotal pancreatectomy. Complete pancreatectomy was therefore performed, conserving the duodenum and the choledochus. Hyperinsulinism thus gave way to diabetes requiring insulin therapy. The child is now 20 months old. Substitution therapy with insulin and Pankreon has allowed normal development.
\end{abstract}

Key words: Nesidioblastosis - Total pancreatectomy.

Zusammenfassung. Zur stationären Aufnahme kam ein Neugeborenes mit therapieresistenten Hypoglycämien bei Hyperinsulinismus. Differentialdiagnostisch konnte eine Nesidioblastose abgegrenzt werden. Nach dem primären Versuch einer subtotalen Pankreatektomie persistierte der Hyperinsulinismus. Deshalb wurde eine totale Pankreatektomie unter Erhaltung des Duodenums und des Ductus choledochus durchgeführt. Ein insulinpflichtiger Diabetes trat an die Stelle des Hyperinsulinismus. Zwischenzeitlich ist das Kind 20 Monate alt. Unter Insulin und Pankreon ${ }^{\mathrm{N}}$ Substitution ist eine normale geistige und körperliche Entwicklung zu beobachten.

Schliisselwörter: Nesidioblastose - Totale Pankreatektomie.

\section{Heterotope Pankreastransplantation bei der Ratte. Endokrine Funktion langzeituiberlebender Transplantate im syngenen Modell}

\section{J. Limmer}

Zentrum für Chirurgie des Klinikum der Universität Ulm, Steinhövelstraße 9, D-7500 Ulm

\section{Heterotopic Pancreas Transplantation in the Rat}

Summary. Transplantation of the vascularized pancreas can yield a long-lasting improvement in streptocotocininduced diabetes in the rat. In syngeneic rats $(n=15)$ rendered diabetic by a single dose of streptocotocin $(50 \mathrm{mg} / \mathrm{kg}$ body weight, i. v.) the whole of the pancreas was transplanted heterotopically, with free intraperitoneal duct drainage. Weight, blood sugar and plasma insulin levels and results of i.v. glucose tolerance tests were monitored. The blood sugar returned to normal levels within 2 days after surgery and remained normal for up to 40 weeks. i.v. glucose tolerance tests showed $\mathrm{K}$-values in the range of $1.6 \%-2.8 \%$ at 25 weeks and of $1.8 \%-3.2 \%$ at 40 weeks.

Key words: Pancreas transplantation, heterotopic.

Zusammenfassung. Diese Studie zeigt die erfolgreiche Therapie eines Diabetes mellitus durch die Transplantation von vascularisiertem Pankreas über langen Zeitraum. Induktion eines Diabetes mellitus bei der Ratte durch die Gabe von Streptocotocin $(50 \mathrm{mg} / \mathrm{kg} / \mathrm{Kg}$; i.v.). Nach Manifestation des Diabetes mellitus erfolgt die heterotope Transplantation des gesamten vascularisierten Pankreas mit freier Drainage des exokrinen Teils in die Bauchhöhle. Kontrolle von Gewicht, Blutzucker, i.v. Glucosetoleranz-Test und Plasmainsulinspiegel. Die Ergebnisse zeigen Normalisierung der Blutzuckerwerte innerhalb 2 Tagen postop. und weiteren Normalverlauf bei über 42 Wochen. K-Werte liegen nach 25 Wochen postop. zwischen 1,6 bis $2,8 \%$, nach 40 Wochen zwischen 1,8 und $3,2 \%$.

Schliusselwörter: Pankreastransplantation, heterotop. 